\title{
Características que definen el fenómeno del botellón en universitarios y adolescentes
}

\author{
María Teresa Cortés Tomás*; Begoña Espejo Tort**; José Antonio Giménez Costa*** \\ * Profesora Titular de Universidad. Dpto. de Psicología Básica. Universitat de Valencia. \\ * Profesora Titular de Universidad. Dpto. de Metodología de las CC. del Comportamiento. Universitat de Valencia. \\ *** Profesor Asociado. Dpto. de Psicología Básica. Universitat de Valencia. \\ Enviar correspondencia a: \\ Maa Teresa Cortés Tomás. Departamento de Psicología Básica. Facultad de Psicología. Universitat de València. Avda. Blasco Ibáñez, 21. 46010 Valencia. Correo \\ electrónico: Maria.T.Cortes@uv.es \\ Recibido: Febrero de 2007 \\ Aceptado: Julio de 2007
}

\section{RESUMEN}

Se presentan algunos de los principales parámetros del patrón de conducta de los jóvenes cuando realizan botellón, intentando superar la visión poco precisa que han generado los estudios de campo. Se han recogido 4083 encuestas de jóvenes entre 14-25 años de la ciudad de Valencia, durante el curso académico 2005/2006 a partir de una muestra aleatoria de centros educativos, acudiendo a las aulas y solicitando la colaboración anónima y voluntaria.

Los resultados confirman la reducción progresiva en la edad de inicio en la práctica del botellón, situándose en 13 años para los adolescentes que en estos momentos tienen 14 años. Asimismo niegan que se trate de una práctica mayoritaria entre varones, al realizarla por igual ambos sexos. También se confirma la tendencia europea que muestra una radicalización del consumo: incremento notorio de bebidas de alta graduación independientemente de la edad y del sexo, llegando a tasas de alcoholemia medias de aproximadamente 1,5 gr., con una frecuencia media de 2 veces por semana y durante más de 6 meses al año. Todo esto suele unirse a una falsa percepción sobre los efectos derivados del consumo al sobredimensionar los aspectos positivos.

Estos resultados plantean la necesidad de diseñar una prevención selectiva o incluso indicada -para aproximadamente el $20 \%$ de casos más extremos-, superando la limitación actual de prevención universal. Tampoco puede obviarse la intervención sobre la permisividad mostrada por los padres fundamentada en aspectos como la edad temprana de inicio en la práctica del botellón o la hora de vuelta a casa tras su realización.

Palabras clave: botellón, patrón de conducta, adolescentes, universitarios.

\section{ABSTRACT}

This paper presents some of the main parameters in the behaviour pattern of young people in their binge drinking practices, seeking to improve the somewhat inaccurate view emerging from field studies. A total of 4,083 questionnaires were administered to young people in Valencia aged 14-25 during the academic year 2005/2006, from a random sample of schools and other educational institutions. The survey was conducted in the institutions themselves and participation was anonymous and voluntary.

The findings confirm a progressive reduction in the age at which this practice begins, 13 having been the starting age for those adolescents who are currently 14 years old. Likewise, the results contradict the assertion that binge drinking is practised mainly by boys, as it is found to an equal extent in either sex. The European trend towards radicalisation of consumption is also confirmed: a considerable increase in the use of drinks with high alcohol content regardless of age and gender, resulting in mean blood alcohol levels of $1.5 \mathrm{gr}$. twice a week on average for more than 6 months a year. All of this is commonly combined with a misleading perception of the effects of alcohol consumption, the positive aspects being overestimated.

These results underline the need to design selective or even indicated prevention programmes for approximately $20 \%$ of the most extreme cases, with a view to overcoming the current constraints of universal prevention. Nor should interventions overlook the issue of parental permissiveness -reflected in aspects such as early age of onset of bingedrinking or lack of controls on the time young people come home after partying.

Key words: binge drinking, behaviour pattern, adolescents, university students. 


\section{INTRODUCCIÓN}

$\mathbf{L}$ a imagen que en la actualidad se tiene del fenómeno del botellón está fuertemente influenciada por lo que transmiten los medios de comunicación. Una lectura rápida de la información que llega a la sociedad suele limitar el botellón a tres aspectos: problemas-quejas vecinales por el ruido o la suciedad generada; el desacuerdo por parte de algunos empresarios con la aplicación de normas que tratan de contrarrestar las demandas vecinales, y finalmente una insistencia generalizada de responsabilizar a la Administración -local, autonómica o nacional- como generadora de soluciones (Pardo, Plaza, Giménez y Cortés, 2005; Plaza, Pardo, Cortés, Samper, Dolz y Martínez, 2006). Además, todo este panorama suele percibirse desde una actitud permisiva y ambivalente ante el consumo de alcohol, que lleva a asumir esta práctica como algo transitorio que ha realizado la mayoría de los españoles cuando eran jóvenes. Pero lo cierto es que los patrones actuales de consumo de los jóvenes durante el botellón difieren, por diversos motivos -cantidad, frecuencia, tipo de alcohol, edad en la que se realizan...-, de los que se daban hace años. Precisamente es este patrón de consumo el que justifica la necesidad de atender a aspectos relacionados con la salud de estos jóvenes, sin dejar de lado las consecuencias de tipo social u orden público a las que se ha aludido anteriormente.

Evidentemente, cualquier alternativa viable ante el botellón no puede limitarse a controlar las consecuencias sociales, sino que debe atender a todo el fenómeno en sí, incluyendo tanto los antecedentes de la conducta -educación por parte de los padres en temas de alcohol y en la ocupación del ocio...-, la propia realización del botellón -motivos, expectativas, bebida consumida, hora de inicio y llegada a casa..., así como las posibles consecuencias derivadas de la misma -embriaguez, accidentes de tráfico, quejas de vecinos, bajo rendimiento académico, vandalismo, relación con otras sustancias adictivas, etc.

Hasta el momento, la mayoría de los estudios empíricos efectuados se han limitado al segundo de estos apartados y han consistido en investigaciones de campo, realizadas durante pocos fines de semana, en las que se ha entrevistado a los jóvenes en las propias zonas botellón. El procedimiento seguido en estas investigaciones genera algunos problemas que es importante considerar. Por una parte, es cuestionable la representatividad de las muestras entrevistadas, ya que la asistencia al botellón está condicionada por factores diversos que en la mayoría de los casos no se han tenido en consideración -exámenes, alguna actividad de ocio especial como un concierto, etc. Asimismo, el número de zonas en las que se han llevado a cabo las entrevistas y el intervalo temporal durante el que se analiza la conducta no han seguido un criterio objetivo y razonado, por lo que los resultados obtenidos probablemente ofrezcan una imagen distorsionada del botellón. Además, al diferir las condiciones bajo las que se han realizado los diferentes estudios, no pueden compararse sus resultados.

Por este motivo, afirmaciones como que un $46.3 \%$ de escolares de San Sebastián entre 12 y 19 años realizan botellón (Elzo, Laespada y Pallarés, 2003) deben tomarse con cautela, pues parecen contradecir otros resultados como los de la Comunidad Valenciana donde se reduce la incidencia a un $16.5 \%$ de escolares entre 14 y 18 años (Huguet, 2007), o las conclusiones a las que llegan Calafat, Juan, Becoña, Castillo, Fernández, Franco et al. (2005), al comparar los resultados de algunos investigadores, afirmando que aunque en el botellón participan miles de jóvenes, a nivel porcentual es una opción de diversión minoritaria que supone alrededor de un $10 \%$. Tampoco parece estar clara la edad a la que suele realizarse el botellón, ya que según el estudio al que se acuda se obtienen unos datos u otros. Mientras GIESYT (2002) afirma que alrededor de un $30 \%$ de los entrevistados tienen entre 14 y 18 años y un 46\% entre 18 y 23 años, otros estudios más recientes realizados en Badajoz y Mérida (Alternativajoven, 2006a, 2006b) parecen confirmar que se trata de una práctica realizada por mayores de 18 años. En ambos estudios el intervalo entre 18 y 25 años es el más representado -Badajoz: 89.2\%; Mérida: $83.2 \%$-. Si se considerase este último dato de manera aislada, podría afirmarse erróneamente que el botellón está descendiendo entre los adolescentes. Nada más lejos de la realidad como ya apuntó Navarrete en 2004, y como se demostrará posteriormente en este trabajo. Posiblemente esta variabilidad en las edades se deba a que las entrevistas se han realizado en una franja horaria diferente, dato que no se especifica en los estudios, pero que puede entreverse a partir de la información relacionada con la hora de inicio y la hora de finalización del botellón. En Extremadura se habla de un intervalo de acceso comprendido entre las 23h. y la $1 \mathrm{~h}$. de la madrugada, mientras que la finalización oscila entre la $1 \mathrm{~h}$. de la madrugada y las $3.30 \mathrm{~h}$., permaneciendo en el botellón una media de 2.5 horas (GIESyT, 2001). Estos datos parecen ser más conservadores en comparación con los obtenidos en otros estudios más recientes, donde se habla de un intervalo entre las $0 \mathrm{~h}$. y las $5 \mathrm{~h}$. de la madrugada (Navarrete, 2004). Pero, nuevamente hay que aludir al modo en que se ha recogido la información, ya que en este último caso no se especifica si la hora de vuelta a casa se refiere a la salida de la zona botellón o si se da por supuesto que todos los jóvenes continúan la noche.

En cuanto a las diferencias por sexo en la asistencia al botellón, a nivel general se alude a un predominio de los varones (Calafat et al., 2005), apoyándose en datos empíricos como los de la investigación de Extremadura, que registran un $58 \%$ de varones frente 
al $41 \%$ de mujeres (GIESyT, 2001). No obstante, las diferencias desaparecen al considerar a los menores de 17 años (GIESyT, 2001, 2002; Navarrete, 2004).

Un dato en el que coinciden todos los estudios analizados es el elevado porcentaje de jóvenes que consumen alcohol durante el botellón, entre un 7090\% de los entrevistados (Alternativajoven, 2006a, 2006b; Baigorri, Fernández y GIESyT, 2003; Calafat et al., 2005; Navarrete, 2004; Nogués, Borrás, Segura, Ruiz y Botella, 2004). Esto viene a corroborar que para la mayoría de los jóvenes el consumo de alcohol y el botellón van unidos.

En cuanto al tipo de bebida que suele consumirse aparecen diferencias en función de la localidad analizada, del momento temporal en el que se ha realizado el estudio y de la edad de los entrevistados. En la parte de Extremadura suele consumirse principalmente combinados de whisky, ginebra o ron (84\%), en comparación con otras bebidas de menor graduación como cerveza (4\%) (GIESyT, 2001, 2002). Las bebidas más representativas de los botellones de Galicia y Jaén son los combinados, el calimocho y la cerveza. Únicamente los madrileños ingieren en mayor porcentaje fermentados (Navarrete, 2004). En todos los casos, se muestran diferencias en función de la edad y el sexo. Por lo general, con la edad se incrementa la ingesta de bebidas destiladas y cerveza y desciende el uso de calimocho, siendo los varones los que suelen consumir más bebidas de alta graduación (GIESyT, 2001; Navarrete, 2004).

No se dispone de información sobre la cantidad de alcohol que se consume durante la realización del botellón. Si se atiende a los resultados publicados en el último Informe Europeo sobre consumo de alcohol entre adolescentes de 15-16 años, en los que se consideran datos ofrecidos por el Plan Nacional Sobre Drogas (PNSD) junto con datos del resto de países (Anderson y Baumberg, 2006), se estima que los jóvenes del sur de Europa, entre los que se incluye España, consumen un equivalente a 40 gramos de alcohol, siendo los varones los que beben mayor cantidad (Anderson y Baumberg, 2006; Navarrete, 2004).

Otro indicador determinante del nivel de consumo es el relacionado con el número de intoxicaciones etílicas que llegan a alcanzarse al realizar el botellón. Según Navarrete (2004) los adolescentes entrevistados informan haber experimentado dos intoxicaciones durante el mes anterior a la realización del estudio, siendo mayor la incidencia entre los varones y los entrevistados de más edad -17 años-. Pero en este caso es necesario advertir que la información sobre alcanzar o no la borrachera puede estar sesgada por la percepción del propio joven, el cual puede no identificar claramente un estado de embriaguez, sobredimensionándolo, o por el contrario no reconociéndolo.
Un aspecto crucial que ofrece una idea aproximada del nivel de implicación de estos jóvenes en el proceso adictivo consiste en analizar la regularidad con la que se efectúa esta conducta, relacionándola con el consumo. Sobre este aspecto se dispone de poca información extraída con muestra española. Concretamente, en los estudios realizados por Navarrete (2004) con adolescentes entre 14 y 17 años, tan sólo se informa de que alrededor del $66 \%$ de los 300 encuestados en Galicia acude todos los fines de semana a hacer botellón, frente al $80.7 \%$ de los 102 encuestados en Jaén y al 89.3\% de los 424 jóvenes madrileños. Una práctica similar al botellón, tanto en cantidad de alcohol que suele ingerirse como en el número de días que se realiza a la semana, es identificada en el informe Alcohol in Europe (2006) como binge drinking. Concretamente, se considera binge drinking el consumo superior a cierto nivel, 5 o más consumiciones, y en una única ocasión (WHO, 2004, en Anderson y Baumberg, 2006). Bajo esta perspectiva europea se estima que aproximadamente un $18 \%$ de los adolescentes de 15-16 años, entre los que están representados jóvenes españoles, incurre en un atracón o consumo de más de 5 copas, tres o más veces en el último mes. Este dato sugiere la necesidad de comprobar, tal como parece indicarse a partir de los datos europeos, si efectivamente se trata de una práctica regular y vinculada en algunos jóvenes a consumos elevados de alcohol.

En cuanto a la frecuencia con la que realizan botellón, hay un claro predomino del fin de semana, incluyendo el jueves y viernes, aunque cabe hacer algunas matizaciones. Por ejemplo, en Extremadura el jueves acude un $18 \%$ de jóvenes, el viernes un 31\% y el sábado supera el 90\% (GIESyT, 2001). No aparecen diferencias respecto al sexo, aunque sí en función de la edad, ya que los jueves la proporción de universitarios es mayor que la de adolescentes, invirtiéndose esta tendencia los sábados, que es claramente el día de asistencia masiva de estudiantes de ESO y Bachillerato (97\%) (GIESyT, 2001).

En general, el 70\% lo hace al menos un día a la semana, preferentemente viernes o sábado, aunque en este caso también oscila entre el $90 \%$ que presenta Mérida y el $60 \%$ de Cáceres. Algo más de la tercera parte de los entrevistados pueden considerarse botelloneros intensivos, ya que hacen botellón siempre que salen o al menos dos días a la semana. En ambos casos, la proporción de varones es claramente superior a la de mujeres (GIESyT, 2001).

La revisión efectuada permite concluir que a pesar de la visión parcial e incompleta que ofrece la investigación actual sobre el botellón, ésta ha sido importante para poder elaborar una primera impresión sobre el fenómeno. Sin embargo, la necesidad actual de fundamentar posibles alternativas ante esta práctica, dada la reducida efectividad de las existentes, requiere dis- 
poner de un conocimiento más detallado, profundo y comprehensivo de todos los aspectos relacionados con la misma. Con este objetivo se ha realizado una amplia investigación que analiza todo el fenómeno del botellón -antecedentes, propia conducta y consecuentes- desde diferentes perspectivas -jóvenes, padres, Fuerzas y Cuerpos de Seguridad y medios de comunicación-.

Debido a las restricciones de los estudios de campo que han servido de base para analizar qué ocurre en el botellón, es lógico que se le conceda la misma importancia a verificar lo poco conocido como a analizar nueva información. Ambos resultados son determinantes para alcanzar una visión más sólida del fenómeno que permita extraer pautas concretas que puedan guiar el diseño o remodelación y ajuste de las alternativas frente al botellón.

En este trabajo, tan sólo se presentan datos referidos al colectivo juvenil entrevistado, abordándose únicamente variables que describen su propia conducta durante el botellón. Esto nos permitirá disponer de un conocimiento más realista y amplio sobre su situación y a partir del mismo, ofrecer unos primeros apuntes que faciliten la configuración de posibles alternativas dirigidas a este colectivo para reducir el fenómeno del botellón. Concretamente, se analizan las características que definen al joven que suele acudir al botellón -sexo, edad, edad de inicio en el consumo de alcohol y edad de inicio en la práctica del botellón-, su patrón de consumo de alcohol, la regularidad con la que suele realizar esta conducta y el intervalo temporal durante el que la lleva a cabo.

Esta investigación supone una mejora respecto a los precedentes ya que se ha contado con una muestra más amplia, entrevistada fuera de las zonas botellón en un ambiente más controlado y con una metodología más rigurosa que ha permitido dejar de lado consideraciones poco fundamentadas o incluso populares.

\section{MÉTODOS}

\section{Instrumento de medida}

Tras revisar los estudios efectuados sobre botellón en España (Aguilera, 2002; Elzo, Laespada y Pallarés, 2003; GIESyT, 2001; 2002; Navarrete, 2004; Nogués et al., 2004; Sánchez y Rubio, 2001) y, por semejanza, sobre el binge drinking europeo (Anderson y Baumberg, 2006), se elaboró una encuesta dirigida a los jóvenes en la que se recogen diferentes aspectos relacionados con la práctica del botellón (edad de inicio, ingesta de alcohol, regularidad de la conducta, motivos, expectativas, opinión sobre las alternativas al botellón, etc.), con cuestiones relacionadas con el ocio (qué actividades hacían antes, cuáles comparten con sus amigos del botellón...) y su percepción sobre la disciplina familiar (consumo que perciben en sus padres, la conducta de sus padres ante el hecho de que ellos realicen botellón, etc.).

\section{Procedimiento}

Se realizó un muestreo estratificado de la población de estudiantes de la Universitat de València y otro muestreo estratificado de la población de estudiantes de centros de Educación Secundaria Obligatoria (ESO), Bachillerato y Ciclos Formativos (CF) de la ciudad de Valencia.

En el caso de la muestra universitaria, se seleccionó una licenciatura o diplomatura de cada área de conocimiento (CC. Básicas, CC. Sociales, CC. de la Salud, Humanidades y CC. de la Educación) con mayor número de matriculados y respondió el cuestionario el grupo más numeroso de cada uno de los cursos.

En cuanto a la muestra no universitaria, se seleccionó aleatoriamente un centro público y otro concertado de cada uno de los 16 distritos educativos que incluyera los estudios de $3^{\circ}$ y $4^{\circ}$ de ESO, Bachillerato y CF. Cuando en un distrito no se disponía de un centro de estas características, se seleccionaba al azar los centros necesarios hasta que todos los estudios estuvieran representados en la muestra. El cuestionario lo cumplimentaban todos los grupos de estudiantes de cada nivel.

Los pases de cuestionarios se realizaron dentro del curso académico 2005/2006, en las aulas docentes y dentro del horario lectivo (mañana o tarde). Los pases estuvieron asistidos en todos los casos por miembros del equipo de investigación. Estos se encargaban de informar a los jóvenes de lo importante que era contar con su propia visión del fenómeno, de presentar el proyecto en su totalidad (evaluación de jóvenes, padres, policías que intervienen en las zonas, vecinos de las zonas afectadas y medios de comunicación) y de pedir finalmente su colaboración, dejando muy claro que era voluntaria y anónima. No hubo ningún joven que no quisiera participar. Además, previo a la codificación de los cuestionarios, dos de los investigadores los revisaron desestimando 68 de ellos.

\section{Muestra}

En total se ha entrevistado a 4083 jóvenes, de los cuales 1361 son universitarios $-34.6 \%$ varones, $65.4 \%$ mujeres- y 2722 son estudiantes de $3^{\circ}$ y $4^{\circ}$ de ESO, Bachillerato y CF $-47.6 \%$ varones y $52.4 \%$ mujeres-. Las edades de los universitarios (U) oscilan entre 19 y 29 años (Media=21.59 años; D.T.=2.27) y las de los adolescentes $(A)$ entre 14 y 18 años (Media=16.16 años; D.T.=1.17). La mayoría de los jóvenes entrevista- 
dos se dedican con exclusividad a estudiar ( $U=73.8 \%$; $A=95.1 \%)$ y residen en el domicilio familiar ( $U=70.4 \%$; $A=99.1 \%)$.

\section{Variables}

A continuación se presentan las variables estudiadas, dentro de cada uno de los bloques de análisis que se han establecido.

\section{Quiénes realizan la conducta y cuándo se presenta}

Se ha medido si los jóvenes han consumido alcohol en alguna ocasión o no, y si realizan botellón o no. Además se han obtenido las edades de inicio en el consumo de alcohol y en el botellón.

\section{Ingesta de alcohol}

En este apartado se ha medido el consumo de alcohol preguntándoles a los jóvenes por el tipo de bebida que suelen consumir -sus respuestas incluían bebidas genéricas (p.e. vodka, ginebra, cerveza...) o marcas comerciales-y por el número de consumiciones que realizan de cada una de las bebidas señaladas.

El tipo de bebida consumida ha sido posteriormente recodificado por los investigadores en tres variables en función de su graduación alcohólica: graduación baja $\left(5^{\circ}-15^{\circ}\right)$, graduación media $\left(16^{\circ}-25^{\circ}\right)$ y graduación alta (más de $30^{\circ}$ ). A partir de esta codificación, se ha clasificado a los entrevistados como consumidores o no de cada uno de estos tipos de bebida. Hay que advertir que un mismo entrevistado puede estar incluido en una, en dos o en las tres variables, ya que estas no se consideran excluyentes. Por ejemplo, un joven que indica que consume cerveza y vodka puntuaría en la variable de graduación baja y también en la de graduación alta.

Además, se ha generado una nueva variable a partir de la suma de las tres anteriores denominada Fuerza de la Graduación Alcohólica de las Bebidas. En ella se diferencian siete niveles: consumo único de un tipo de bebida, consumo combinado de dos tipos, y consumo de los tres tipos establecidos. En este caso las categorías son excluyentes, ya que por ejemplo un entrevistado puede consumir sólo bebidas de graduación alta -p.e. vodka, ginebra...- lo que lo diferenciaría de otro que combinara bebidas de graduación alta y baja -p.e. ginebra y cerveza-.

También se ha medido el número de borracheras que dicen haber experimentado en el último mes previo a la realización de la encuesta, y si tenían intención de llegar a este estado o no.

\section{Regularidad de la conducta}

En este apartado se ha medido la probabilidad de ocurrencia del botellón en cada uno de los meses del año, desde que se inició esta conducta. Se ha utilizado una escala de respuesta tipo Likert con cuatro puntos con los siguientes anclajes verbales: 1. "Nunca suelo hacer botellón en el mes de...", 2. "A veces he hecho botellón durante el mes de...", 3. "A menudo hago botellón en el mes de ..." y 4. "Siempre hago botellón en el mes de ...".

También se ha preguntado por la frecuencia semanal con la que suelen realizar el botellón. En este caso se diferenciaba día a día, utilizando la misma escala de respuesta.

También se ha medido el número de meses al año que realizan botellón y el número de días a la semana que lo practican, independientemente de la frecuencia, así como el número de años que llevan realizando la conducta.

\section{Intervalo temporal de la conducta}

En este caso se ha considerado la hora de llegada al lugar donde se realiza botellón, la hora de salida del mismo y el número de horas durante las que se realiza la conducta.

\section{Análisis}

Todos los análisis han sido realizados mediante el paquete estadístico SPSS 14.0 para Windows. A continuación se detallan los análisis realizados para cada uno de los bloques considerados.

\section{Quiénes realizan la conducta y cuándo se presenta}

Se ha medido si los jóvenes consumen alcohol o no, y si realizan botellón o no, obteniendo los porcentajes correspondientes, y se ha contrastado si existen diferencias entre universitarios y adolescentes en ambas variables mediante sendas pruebas $\chi^{2}$. Asimismo, se ha contrastado la existencia de diferencias entre varones $(\mathrm{V})$ y mujeres $(\mathrm{M})$ en el consumo de alcohol, y en la realización de botellón, mediante las correspondientes pruebas $\chi^{2}$. Estas pruebas se han realizado en adolescentes y en universitarios.

Para comprobar si existen diferencias significativas en la edad de inicio en el consumo de alcohol, y en la edad de inicio en la práctica del botellón, en función del grupo (U-A) y del sexo (V-M), se ha realizando dos análisis de varianza (ANOVA) factoriales entresujetos.

Además, puesto que los adolescentes constituyen la población sobre la que ha de planificarse las medidas preventivas, se ha estudiado si existen diferencias significativas en el inicio en el consumo de alcohol en 
función de la edad actual de dichos jóvenes (14 a 18 años), con objeto de comprobar si hay un descenso en la edad de inicio cuanto más jóvenes son. Para ello se ha realizado un ANOVA unifactorial entre sujetos, en el que la variable dependiente (VD) es la edad de inicio en el consumo de alcohol, y la variable independiente (VI) es la edad actual de los jóvenes (cinco niveles). Se ha utilizado el mismo procedimiento para comprobar si aparecen diferencias significativas en el inicio de la práctica del botellón en función de la edad actual de los jóvenes (14 a 18 años), pero considerando como VD la edad en que se realizó el primer botellón. La prueba a posteriori utilizada ha sido Games-Howell, ya que no existía homogeneidad de varianzas.

Por último, se ha contrastado la proporción de universitarios que se iniciaron al mismo tiempo en el consumo de alcohol y en el botellón, frente a la proporción de adolescentes que también se iniciaron a la vez en ambas conductas, utilizando pruebas de contraste de proporciones para grupos independientes.

\section{Ingesta de alcohol}

Tras calcular el porcentaje de jóvenes que consumen alcohol durante el botellón, se ha realizado un ANOVA factorial entresujetos para comprobar si el número de copas consumidas (VD) difiere en función del grupo (U-A) y del sexo (V-M).

Para comprobar si adolescentes y universitarios consumen el mismo tipo de bebidas se ha realizado una prueba $\chi^{2}$ para cada una de las tres variables creadas (bebidas de graduación baja, graduación media y graduación alta) por separado. Además, y para cada grupo (U-A), se ha comprobado si existen diferencias en el tipo de consumo en función del sexo (V-M), realizándose también pruebas $\chi^{2}$ para cada una de las tres variables creadas.

Para comprobar si hay diferencias en función del grupo (U-A) en la Fuerza de la Graduación Alcohólica de las Bebidas que consumen se ha realizado una prueba $\chi^{2}$.

Se ha obtenido el porcentaje de jóvenes que afirma haberse emborrachado alguna vez, así como el promedio de borracheras para toda la muestra. También se ha realizado un ANOVA factorial entresujetos considerando como variables independientes el sexo (V-M), el grupo (U-A) y la intención de emborracharse (Sí-No), para ver su influencia sobre el número de borracheras (VD).

\section{Regularidad de la conducta del botellón}

Respecto a la regularidad a lo largo del año, en primer lugar se ha calculado los porcentajes de frecuencia mensual con que hacen botellón los adolescentes y los universitarios. Además se ha contabilizado el número de meses que lo hacen. En cuanto a la regularidad semanal, se ha calculado los porcentajes de frecuencia semanal con que hacen botellón adolescentes y universitarios.

Por otra parte, se ha calculado los porcentajes de frecuencia mes a mes en función de la frecuencia semanal (uno, dos o tres días). También se ha calculado los porcentajes de frecuencia mensual a lo largo de todo el año en función de la cantidad de alcohol ingerida (1-2 copas, 3-4 copas, 5-6 copas, 7 ó más copas). En ambos casos los cruces se han realizado para universitarios y para adolescentes.

Asimismo, se ha calculado cómo se distribuyen los jóvenes de cada grupo (U-A) en función del número de años que llevan haciendo botellón, así como el promedio de años que llevan realizando la conducta.

Por último, se ha comprobado, si el número de años que los jóvenes llevan haciendo botellón se relaciona con diversas variables. Para ello, se ha llevado a cabo un ANOVA factorial entresujetos considerando como VD los años que llevan haciendo botellón, y como VIs el grupo (U-A), el sexo ( $\mathrm{V}-\mathrm{M})$ y el consumo de alcohol (1-2 copas, 3-4 copas, 5-6 copas, 7 ó más copas).

\section{Intervalo temporal de la conducta}

En este caso se ha calculado el promedio de hora de llegada al lugar donde se realiza botellón, así como el promedio de hora de salida del mismo.

Para comprobar si existen diferencias significativas en estas horas en función del grupo y del sexo se ha realizado dos ANOVAs entresujetos, uno considerando como VD la hora de llegada, y otro considerando como VD la hora de salida. En ambos casos las VIs han sido el grupo (U-A) y el sexo (V-M).

Por último, se ha realizado un ANOVA entresujetos para comprobar si existen diferencias en el número de horas durante las que realizan botellón (VD) en función de tres VIs: el sexo (V-M), el grupo (U-A) y el consumo de alcohol (1-2 copas, 3-4 copas, 5-6 copas, 7 ó más copas). La prueba a posteriori utilizada ha sido Games-Howell, ya que no existía homogeneidad de varianzas.

\section{RESULTADOS}

\section{Quiénes realizan la conducta y cuándo se presenta}

El $97.7 \%$ de los universitarios $(n=1329)$ ha consumido alcohol en alguna ocasión. Entre los adolescentes $(n=2406)$ este porcentaje es significativamente menor $\left(88.7 \%, \chi^{2}=96.02, \mathrm{p}<0.001\right)$. En universitarios 
la edad promedio de inicio en el consumo de alcohol se sitúa en los 14.5 años (D.T.=1.87) mientras que en adolescentes hablamos de una edad promedio de inicio de 13.3 años (D.T.=1.99).

Por otra parte, el $71.9 \% \quad(n=2917)$ de los jóvenes entrevistados realiza botellón. De ellos, el 80.6\% $(n=1094)$ son universitarios y el 67\% ( $n=1823)$ adolescentes, siendo significativamente mayor la proporción de universitarios que hacen botellón $\left(\chi^{2}=80.58\right.$, $p<0.001)$. En universitarios la edad promedio de inicio en el botellón se sitúa en los 15.5 años (D.T.=1.8) mientras que en adolescentes es de 14.1 (D.T.=1.3) años.

No aparecen diferencias estadísticamente significativas en el número de varones y mujeres que afirman consumir alcohol y haber practicado botellón en alguna ocasión, y eso ocurre tanto en universitarios (consumo: $\chi^{2}=0.005, p<0.94$; botellón: $\chi^{2}=0.06$, $\mathrm{p}<0.80$ ) como en adolescentes (consumo: $\chi^{2}=0.32$, $p<0.57$; botellón: $\chi^{2}=0.64, p<0.43$ )

Sólo se aprecian diferencias significativas en la edad de inicio en el consumo de alcohol en función del grupo (U: Media=14.5; A: Media=13.3; $F_{(1,3651)}=350.33$, $\mathrm{MCE}=4.47, \mathrm{p}<0.001$ ), y lo mismo ocurre con la edad de inicio en el botellón (U: Media=15.5; A: Media=14.1; $\left.\mathrm{F}_{(1,2819)}=611.67, \mathrm{MCE}=2.20, \mathrm{p}<0.001\right)$.

Para comprobar a partir de qué edad actual aparecen diferencias significativas en las edades de inicio en el consumo de alcohol y en el botellón, se han realizado los ANOVAs con los adolescentes (tabla 1). Los resultados indican diferencias significativas tanto en la edad de inicio en el consumo de alcohol $\left(F_{(15,3606)}=40.31, M C E=3.12, p<0.001\right)$, como en la edad de inicio en el botellón $\left(F_{(15,2804)}=71.97\right.$, $\mathrm{MCE}=1.96, \mathrm{p}<0.001)$, confirmando un descenso significativo, año a año. Es decir, los adolescentes de 17 años se han iniciado en el consumo de alcohol a una edad significativamente más temprana que los de 18 años. $Y$ así progresivamente hasta los 15 años en el consumo de alcohol y hasta los 14 años en el boteIlón. En el consumo de alcohol se aprecia una estabilización en la edad de inicio entre los más jóvenes (es similar en los jóvenes de 14 y 15 años), mientras que en el botellón sigue existiendo una disminución progresiva y significativa de la edad de inicio.

Los contrastes de proporciones (tabla 2) confirman que el porcentaje de adolescentes que se iniciaron en ambas conductas al mismo tiempo es significativamente mayor que el de universitarios.

Tabla 1. Edades medias de inicio en el consumo de alcohol y botellón en adolescentes, y resultados de las pruebas a posteriori

\begin{tabular}{|c|c|c|c|c|c|c|c|c|}
\hline & $\begin{array}{l}\text { Vl: Edad } \\
\text { actual } \\
\text { (años) }\end{array}$ & $\mathbf{N}$ & $\begin{array}{l}\text { Edades } \\
\text { medias de } \\
\text { inicio }\end{array}$ & Prok & lades a & $\begin{array}{l}\text { as en I } \\
\text { les-Ho }\end{array}$ & ebas a & riori \\
\hline \multirow{5}{*}{$\begin{array}{l}\text { VD: } \\
\text { Consumo } \\
\text { de alcohol }\end{array}$} & \multirow{5}{*}{$\begin{array}{l}18 \\
17 \\
16 \\
15 \\
14 \\
\end{array}$} & \multirow{5}{*}{$\begin{array}{l}374 \\
627 \\
687 \\
496 \\
154 \\
\end{array}$} & \multirow{5}{*}{$\begin{array}{l}14.17 \\
13.57 \\
13.15 \\
12.54 \\
12.51 \\
\end{array}$} & 14.17 & 13.57 & 13.15 & 12.54 & 12.51 \\
\hline & & & & - & & & & \\
\hline & & & & 0.001 & - & & & \\
\hline & & & & 0.001 & 0.005 & -- & & \\
\hline & & & & 0.001 & 0.001 & 0.001 & $\overline{1}$ & - \\
\hline \multirow{6}{*}{$\begin{array}{l}\text { VD: } \\
\text { Realización } \\
\text { de botellón }\end{array}$} & \multirow{6}{*}{$\begin{array}{l}18 \\
17 \\
16 \\
15 \\
14\end{array}$} & \multirow{6}{*}{$\begin{array}{c}320 \\
511 \\
503 \\
341 \\
76\end{array}$} & \multirow{6}{*}{$\begin{array}{l}14.91 \\
14.38 \\
13.82 \\
13.38 \\
12.99\end{array}$} & 14.91 & 14.38 & 13.82 & 13.38 & 12.99 \\
\hline & & & & - & & & & \\
\hline & & & & 0.001 & - & & & \\
\hline & & & & 0.001 & 0.001 & - & & \\
\hline & & & & 0.001 & 0.001 & 0.001 & - & \\
\hline & & & & 0.001 & 0.001 & 0.001 & 0.05 & -- \\
\hline
\end{tabular}

Tabla 2. Contrastes de proporciones entre universitarios y adolescentes

\begin{tabular}{|c|c|c|c|c|c|c|}
\hline & & \multicolumn{5}{|c|}{ Edad de inicio consumo alcohol / Edad inicio botellón } \\
\hline & & $12 / 12$ & $13 / 13$ & $14 / 14$ & $15 / 15$ & $16 / 16$ \\
\hline \multirow{2}{*}{ Universitarios } & Proporción & 0.24 & 0.39 & 0.38 & 0.40 & 0.48 \\
\hline & N & 79 & 176 & 315 & 203 & 150 \\
\hline \multirow{2}{*}{ Adolescentes } & Proporción & 0.42 & 0.50 & 0.60 & 0.70 & 0.85 \\
\hline & N & 245 & 483 & 468 & 245 & 72 \\
\hline Z & & $2.87^{*}$ & $2.50^{*}$ & $6.04^{*}$ & $6.37^{*}$ & $5.27^{*}$ \\
\hline
\end{tabular}


Tabla 3. Porcentajes de consumo por grupo y sexo

\begin{tabular}{|c|c|c|c|c|c|c|c|}
\hline & \multicolumn{3}{|c|}{ Graduación } & & \multicolumn{3}{|c|}{ Graduación } \\
\hline & Baja & Media & Alta & & Baja & Media & Alta \\
\hline \multirow{2}{*}{ A } & \multirow{2}{*}{$35.9 *$} & \multirow{2}{*}{$72.2^{*}$} & \multirow{2}{*}{82.5} & V & 36.4 & 65.5 & 83.9 \\
\hline & & & & M & 35.3 & $78.1^{*}$ & 81.1 \\
\hline \multirow{2}{*}{$U$} & \multirow{2}{*}{26.4} & \multirow{2}{*}{46.9} & \multirow{2}{*}{79.6} & V & $30.8^{* *}$ & 36.8 & $83.4^{* *}$ \\
\hline & & & & M & 24.1 & $52.3^{* *}$ & 77.7 \\
\hline & \multicolumn{7}{|c|}{ Fuerza de la Graduación Alcohólica de las Bebidas } \\
\hline & Grad. Baja & $\begin{array}{c}\text { Graduación } \\
\text { media }\end{array}$ & Grad. alta & $\begin{array}{l}\text { G. Baja + } \\
\text { G. Media + } \\
\text { G. Alta }\end{array}$ & $\begin{array}{l}\text { G. Baja + } \\
\text { G. Media }\end{array}$ & $\begin{array}{c}\text { G. Baja + } \\
\text { G. Alta }\end{array}$ & $\begin{array}{c}\text { G. Media + } \\
\text { G. Alta }\end{array}$ \\
\hline A & 2.7 & 7.1 & 13.9 & 23.4 & 3.1 & 6.5 & 38.6 \\
\hline U & 3.7 & 9.2 & 36.5 & 11.0 & 3.1 & 8.6 & 23.6 \\
\hline
\end{tabular}

U: Universitarios; A: Adolescentes; V: Varones; M: Mujeres; ${ }^{*} p<0.001 ;{ }^{* *} p<0.05$

\section{Ingesta de alcohol}

Un $82.2 \%$ de los jóvenes que realizan botellón afirman consumir alcohol. Como promedio se consumen durante el botellón unas 5.3 copas (D.T.=2.52), oscilando la ingesta de la mayoría de los entrevistados entre 3 y 6 copas (65.2\% del total). Sólo se aprecian diferencias significativas en el nivel de consumo en función del sexo ( $\mathrm{V}$ : Media=6.46; $\mathrm{M}$ : Media=4.86; $\left.F_{(1,2404)}=128.48, M C E=10.81, p<0.001\right)$, pero no del grupo.

Al comparar el consumo de adolescentes con el de universitarios (tabla 3 ) los resultados indican que los adolescentes consumen más bebidas de graduación baja $\left(\chi^{2}=27.53, p<0.001\right)$ y de graduación media $\left(\chi^{2}=186, p<0.001\right)$, mientras que se consumen por igual bebidas de graduación alta. Al diferenciar por sexo dentro de cada grupo, se observa que los universitarios varones consumen más bebidas de graduación baja $\left(\chi^{2}=5.33, p<0.021\right)$ y alta $\left(\chi^{2}=4.67, p<0.031\right)$, mientras que las mujeres consumen más bebidas de graduación media $\left(\chi^{2}=23.21, p<0.001\right)$. Sin embargo, entre los adolescentes sólo se mantiene esta última diferencia $\left(\chi^{2}=35.16, p<0.001\right)$ y en el mismo sentido.

Respecto a si hay diferencias entre universitarios y adolescentes en la Fuerza de la graduación alcohólica de las Bebidas que consumen (tabla 3), los resultados indican que existen diferencias significativas entre ambos grupos $\left(\chi^{2}=233.99, p<0.001\right)$. Por tanto, aunque ambos grupos no diferían en el consumo de bebidas de graduación alta, a partir de esta prueba puede matizarse que en el caso de los adolescentes se combinan en la mayoría de los casos bebidas de graduación alta con bebidas de menor graduación, mientras que en el consumo de los universitarios el mayor peso lo tiene el consumo de bebidas de alta graduación.
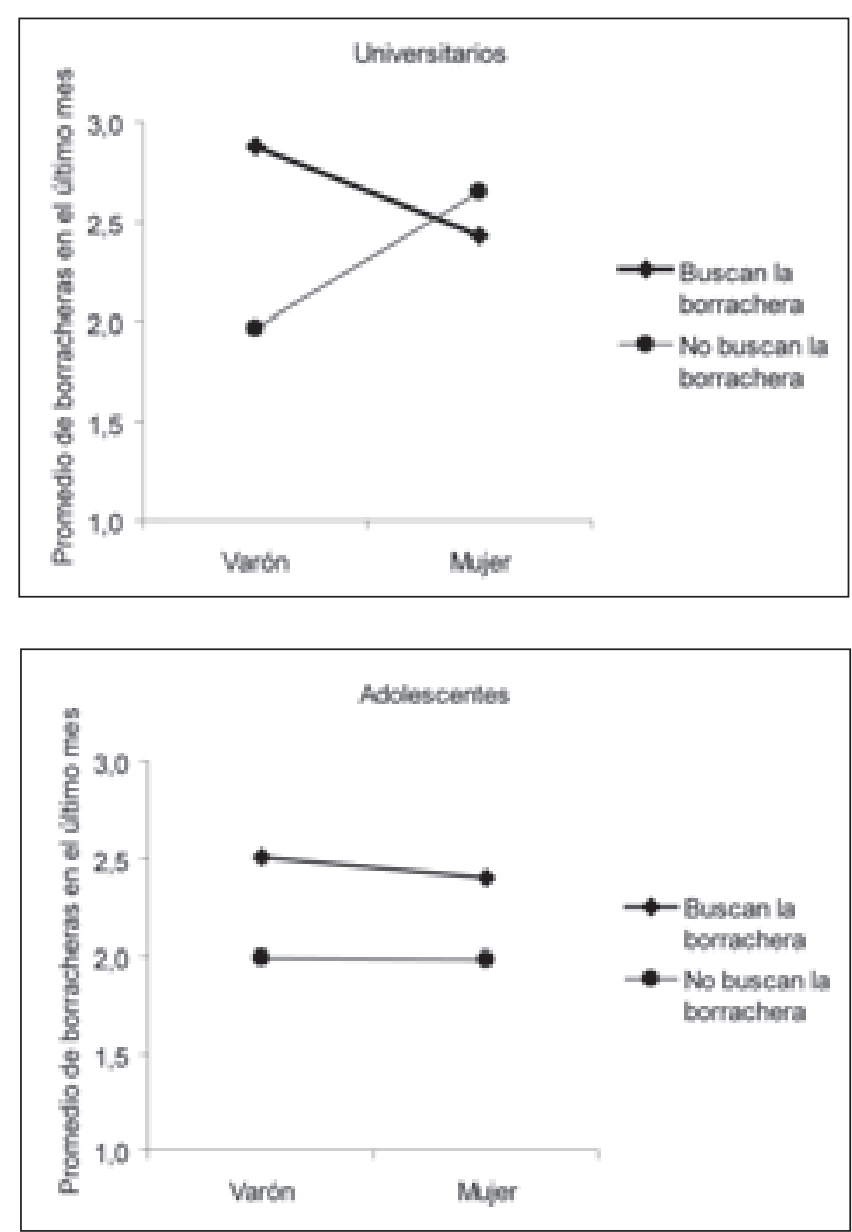

Figura 1. Gráficas para la triple interacción

Del total de jóvenes que hace botellón, el $40.9 \%$ afirma haberse emborrachado alguna vez durante el último mes ( 1 vez $=16.9 \%, 2$ veces $=10.8 \%, 3$ veces $=6.3 \%, 4$ veces $=3.1 \%, 5$ veces o más $=3.8 \%$ ), siendo el promedio de borracheras de 2.37 (D.T.=1.95). 
En cuanto a la influencia del sexo, el grupo y la intención de emborracharse sobre el número de borracheras, los resultados del ANOVA indican que la interacción entre los tres factores es estadísticamente significativa $\left(F_{(1,1069)}=4.25, M C E=15.85, p<0.04\right)$. Como se observa en la figura 1, entre los universitarios, los varones con intención de emborracharse tienen un promedio de borracheras en el último mes (2.9) mucho mayor que los que no la tienen (2), mientras que en las mujeres no se da esta diferencia (Buscan la borrachera=2.4; No buscan la borrachera=2.6). Sin embargo, entre los adolescentes se observa que sí existe una diferencia en el número de borracheras para los que la buscan y para los que no, independientemente del sexo. Es decir, entre los adolescentes, los varones (2.5) y las mujeres (2.4) que buscan la borrachera se emborrachan más veces que los que no la buscan (Varones=2; Mujeres=2).

\section{Regularidad de la conducta del botellón}

Se hace botellón durante todo el año, incrementándose su frecuencia en marzo, abril, verano (junio a septiembre) y diciembre (tabla 4).
Por otra parte, se ha observado que el $83.1 \%$ de universitarios $(n=877)$ y el $72.1 \%$ de adolescentes $(n=1398)$ realiza botellón durante seis meses o más. Concretamente, el $47.4 \%$ de los universitarios $(n=470)$ y el $36.38 \%$ de los adolescentes $(n=650)$ lo hacen todos los meses del año. Por último, como promedio los universitarios realizan botellón 9.71 meses al año (D.T.=2.89), y los adolescentes 8.77 meses (D.T.=3.33).

En cuanto a la frecuencia semanal con que se realiza botellón, los universitarios asisten los jueves, viernes y sábados, mientras que los adolescentes prefieren iniciarse el viernes y mantenerse, con menor frecuencia, hasta el domingo (tabla 5).

Se ha comprobado además si la frecuencia semanal con que se hace botellón varía en función del número de meses en que se realiza esta conducta. Tal como indica la tabla 6, tanto en universitarios como en adolescentes, el porcentaje de jóvenes que realizan botellón menos meses suelen hacerlo una o dos veces a la semana, mientras que quienes hacen botellón más meses, lo hacen de dos a tres veces a la semana.

Tabla 4. Porcentajes de jóvenes que realizan botellón a lo largo del año (porcentajes de jóvenes por columna)

\begin{tabular}{|l|l|c|c|c|c|c|c|c|c|c|c|c|c|}
\hline & & Enero & Febrero & Marzo & Abril & Mayo & Junio & Julio & Agosto & Sept. & Oct. & Nov. & Dic. \\
\hline \multirow{4}{*}{ U } & Nunca & 28.9 & 35.4 & 10.4 & 13.6 & 22.4 & 30.3 & 9.8 & 6.3 & 11.8 & 18.4 & 22.5 & 13.9 \\
& Aveces & $\mathbf{5 5 . 3}$ & $\mathbf{5 2 . 9}$ & $\mathbf{5 1 . 3}$ & $\mathbf{5 6 . 8}$ & $\mathbf{5 5 . 0}$ & $\mathbf{4 0 . 7}$ & $\mathbf{3 9 . 9}$ & $\mathbf{3 6 . 2}$ & $\mathbf{5 1 . 1}$ & $\mathbf{5 8 . 0}$ & $\mathbf{6 0 . 1}$ & $\mathbf{5 2 . 9}$ \\
& A menudo & 12.9 & 9.3 & $\mathbf{2 8 . 1}$ & $\mathbf{2 3 . 7}$ & 17.5 & $\mathbf{2 1 . 7}$ & $\mathbf{3 3 . 9}$ & $\mathbf{3 6 . 8}$ & $\mathbf{2 5 . 9}$ & 18.1 & 13.5 & $\mathbf{2 5 . 7}$ \\
& Siempre & 2.9 & 2.4 & 10.3 & 5.9 & 5.1 & 7.3 & $\mathbf{1 6 . 4}$ & $\mathbf{2 0 . 7}$ & $\mathbf{1 1 . 2}$ & 5.5 & 3.9 & 7.5 \\
\hline \multirow{4}{*}{ A } & Nunca & 30.3 & 42.7 & 13.9 & 28.8 & 37.8 & 21.0 & 10.4 & 7.5 & 24.9 & 37.7 & 40.1 & 17.1 \\
& A veces & $\mathbf{5 2 . 7}$ & $\mathbf{4 6 . 7}$ & $\mathbf{3 4 . 8}$ & $\mathbf{4 7 . 7}$ & $\mathbf{4 5 . 8}$ & $\mathbf{4 1 . 7}$ & $\mathbf{3 2 . 9}$ & $\mathbf{2 8 . 9}$ & $\mathbf{4 5 . 4}$ & $\mathbf{4 7 . 8}$ & $\mathbf{4 6 . 3}$ & $\mathbf{4 4 . 8}$ \\
& A menudo & 13.1 & 8.1 & $\mathbf{3 4 . 1}$ & 17.9 & 12.6 & $\mathbf{2 7 . 3}$ & $\mathbf{3 8 . 7}$ & $\mathbf{3 8 . 1}$ & $\mathbf{2 1 . 3}$ & 11.2 & 10.9 & $\mathbf{2 6 . 3}$ \\
& Siempre & 3.9 & 2.6 & $\mathbf{1 7 . 2}$ & 5.7 & 3.7 & $\mathbf{1 0 . 0}$ & $\mathbf{1 7 . 9}$ & $\mathbf{2 5 . 6}$ & 8.4 & 3.3 & 2.7 & $\mathbf{1 1 . 9}$ \\
\hline
\end{tabular}

U: universitarios; A: adolescentes

Tabla 5. Porcentajes de jóvenes que realizan botellón a lo largo de la semana (porcentajes de jóvenes por columna)

\begin{tabular}{|c|c|c|c|c|c|c|c|c|}
\hline & & Lunes & Martes & Miércoles & Jueves & Viernes & Sábado & Domingo \\
\hline \multirow{4}{*}{ U } & Nunca & 97.8 & 97.7 & 95.6 & 54.4 & 20.0 & 1.5 & 89.5 \\
\hline & A veces & 2.1 & 2.2 & 4.3 & 37.0 & 58.9 & 49.3 & 9.4 \\
\hline & A menudo & 0.1 & 0.1 & 0.1 & 7.3 & 17.0 & 34.5 & 1.0 \\
\hline & Siempre & 0.0 & 0.0 & 0.0 & 1.3 & 4.1 & 14.6 & 0.2 \\
\hline \multirow{4}{*}{ A } & Nunca & 96.8 & 96.6 & 94.6 & 90.5 & 17.9 & 2.7 & 75.2 \\
\hline & A veces & 3.1 & 3.4 & 5.1 & 8.8 & 50.9 & 44.9 & 18.8 \\
\hline & A menudo & 0.1 & 0.1 & 0.3 & 0.6 & 23.6 & 36.5 & 4.7 \\
\hline & Siempre & 0.0 & 0.0 & 0.1 & 0.1 & 7.7 & 15.8 & 1.3 \\
\hline
\end{tabular}

U: universitarios; A: adolescentes 
Tabla 6. Frecuencia semanal con la que se hace botellón en función del número de meses que lo realizan (porcentajes de jóvenes por columna)*

\begin{tabular}{|c|c|c|c|c|c|c|c|c|c|c|c|c|c|c|}
\hline & $\begin{array}{c}\text { Número de días } \\
\text { a la semana }\end{array}$ & & 1 & 2 & 3 & 4 & 5 & 6 & 7 & 8 & 9 & 10 & 11 & 12 \\
\hline \multirow{6}{*}{ U } & \multirow{2}{*}{1} & $N$ & 2 & 8 & 9 & 14 & 12 & 11 & 13 & 11 & 14 & 12 & 5 & 38 \\
\hline & & $\%$ & 50.0 & 61.5 & 37.5 & 35.9 & 33.3 & 21.6 & 26.5 & 13.1 & 20.3 & 12.8 & 6.6 & 7.7 \\
\hline & \multirow{2}{*}{2} & $N$ & 2 & 2 & 13 & 19 & 20 & 27 & 24 & 42 & 31 & 30 & 31 & 204 \\
\hline & & $\%$ & 50.0 & 15.4 & 54.2 & 48.7 & 55.6 & 52.9 & 49.0 & 50.0 & 44.9 & 31.9 & 40.8 & 41.3 \\
\hline & \multirow{2}{*}{3} & $N$ & 0 & 3 & 2 & 5 & 3 & 12 & 12 & 29 & 24 & 45 & 31 & 187 \\
\hline & & $\%$ & 0.0 & 23.1 & 8.3 & 12.8 & 8.3 & 23.5 & 24.5 & 34.5 & 34.8 & 47.9 & 40.8 & 37.9 \\
\hline \multirow{6}{*}{ A } & \multirow{2}{*}{1} & $N$ & 12 & 22 & 31 & 22 & 29 & 24 & 20 & 11 & 8 & 7 & 5 & 42 \\
\hline & & $\%$ & 54.5 & 48.9 & 41.9 & 26.8 & 24.2 & 20.0 & 16.1 & 8.6 & 6.8 & 5.3 & 4.0 & 6.5 \\
\hline & \multirow{2}{*}{2} & $N$ & 5 & 19 & 37 & 48 & 64 & 66 & 73 & 89 & 77 & 94 & 78 & 406 \\
\hline & & $\%$ & 22.7 & 42.2 & 50.0 & 58.5 & 53.3 & 55.0 & 58.9 & 69.5 & 65.3 & 71.2 & 62.9 & 62.5 \\
\hline & & $N$ & 3 & 3 & 3 & 11 & 23 & 26 & 22 & 20 & 29 & 22 & 32 & 135 \\
\hline & & $\%$ & 13.6 & 6.7 & 4.1 & 13.4 & 19.2 & 21.7 & 17.7 & 15.6 & 24.6 & 16.7 & 25.8 & 20.8 \\
\hline
\end{tabular}

U: universitarios; A: adolescentes; * Se han omitido los porcentajes a partir de 4 días o más

Tabla 7. Frecuencia mensual con la que se realiza botellón, en función del consumo (porcentajes de jóvenes por columna)

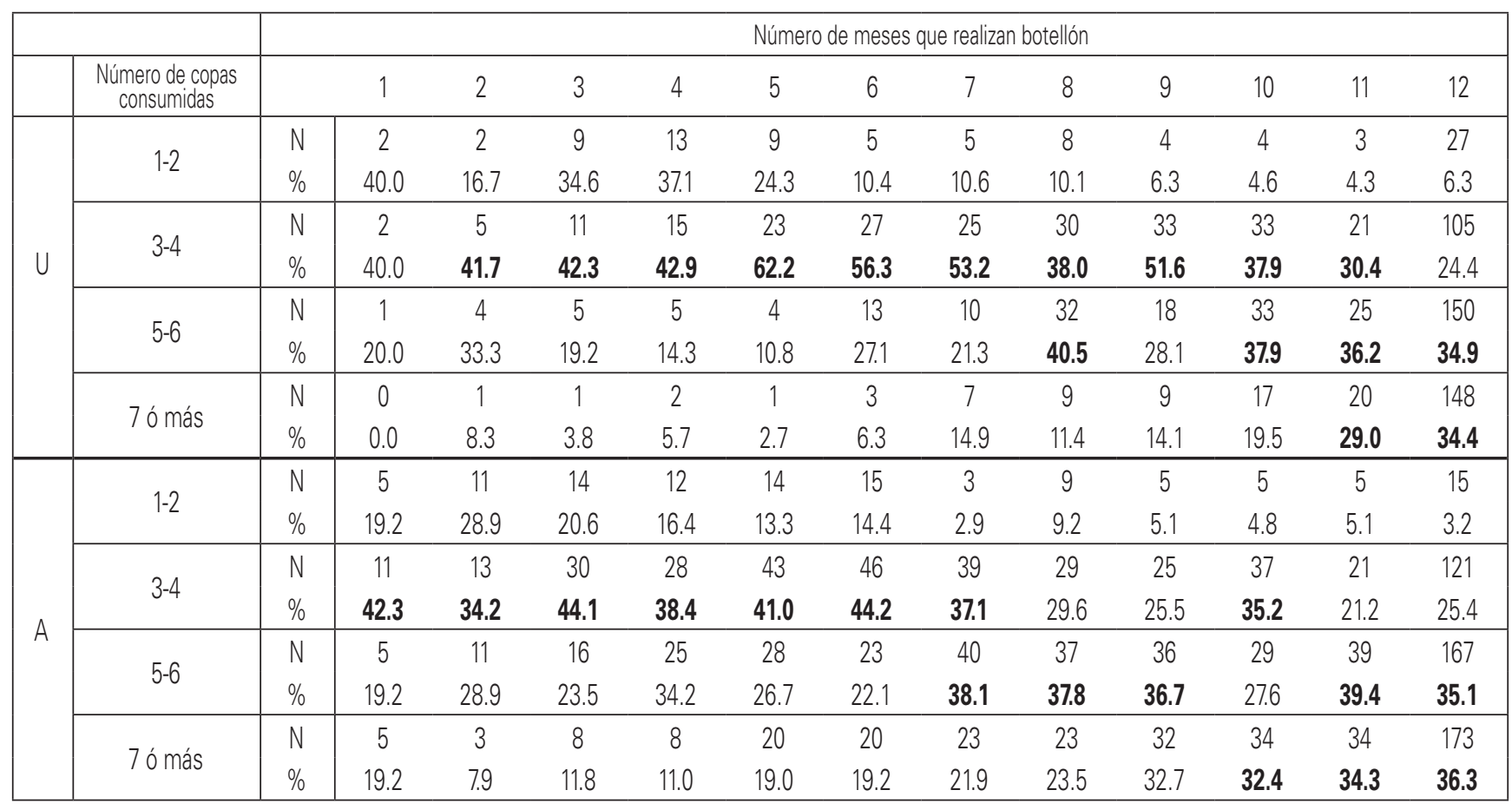

U: universitarios; A: adolescentes

Al tener en cuenta la ingesta de alcohol realizada (tabla 7) se aprecia que, independientemente del grupo, cuanto mayor es el consumo, más meses al año se realiza botellón.

En cuanto al promedio de años que llevan realizando botellón los universitarios, éste se sitúa en 5.64 años (D.T.=2.22), mientras que en los adolescentes desciende a 2.32 años (D.T.=1.27). Además, en la tabla 8 se observa que más de un $5 \%$ de universitarios lleva 10 años o más realizando la conducta. También se observa que un 5\% de adolescentes llevan haciendo botellón desde hace 5 años o más. 
Tabla 8. Años que Ilevan haciendo botellón

\begin{tabular}{|c|c|c|c|c|c|}
\hline \multicolumn{3}{|c|}{ Universitarios } & \multicolumn{3}{|c|}{ Adolescentes } \\
\hline Años & Frecuencia & Porcentaje & Años & Frecuencia & Porcentaje \\
\hline 1 & 11 & 1.1 & $<1$ & 80 & 4.6 \\
\hline 2 & 45 & 4.4 & 1 & 382 & 21.9 \\
\hline 3 & 124 & 12.2 & 2 & 600 & 34.3 \\
\hline 4 & 161 & 15.9 & 3 & 393 & 22.5 \\
\hline 5 & 176 & 17.4 & 4 & 202 & 11.6 \\
\hline 6 & 166 & 16.4 & 5 & 61 & 3.5 \\
\hline 7 & 130 & 12.8 & 6 & 21 & 1.2 \\
\hline 8 & 80 & 7.9 & 7 & 7 & 0.4 \\
\hline 9 & 61 & 6.0 & 8 & 1 & 0.1 \\
\hline 10 & 37 & 3.7 & Total & 1747 & 100 \\
\hline 11 & 19 & 1.9 & & & \\
\hline 12 & 2 & 0.2 & & & \\
\hline 14 & 1 & 0.1 & & & \\
\hline Total & 1013 & 100 & & & \\
\hline
\end{tabular}

Los resultados del ANOVA efectuado para comprobar si el número de años que los jóvenes llevan haciendo botellón varía en función del grupo, el sexo y el consumo de alcohol, indican que sólo existe interacción estadísticamente significativa entre Sexo y Consumo de alcohol $\left(F_{(3,2265)}=4.27, M C E=2.73\right.$, $p<0.005)$. Aunque la interacción entre el Grupo y el Consumo de alcohol no es estadísticamente significativa (figura 2), sí existen diferencias dentro de cada grupo en el número de años que llevan haciendo botellón en función del nivel de consumo (U: $F_{(3,902)}=12.65, M C E=4.69, p<0.001 ; A: F_{(3,1374)}=21.24$, $M C E=1.52, p<0.001)$. Tras realizar las pruebas a posteriori (U: Tukey; A: Games-Howell) para comprobar cómo difiere el consumo de alcohol en cada grupo, los resultados indican que, para universitarios, todas las diferencias son estadísticamente significativas excepto entre los niveles más altos de consumo (5-6 copas, 7 copas o más). Y para los adolescentes todas las diferencias son estadísticamente significativas excepto para los consumos más bajos (1-2 copas, 3-4 copas). Es decir, en general se observa, para ambos grupos, que los jóvenes que más consumen son los que más tiempo llevan haciendo botellón.

En cuanto a los niveles de la interacción entre Sexo y Consumo de alcohol (figura 3) se observa que, tanto entre los varones como entre las mujeres existen diferencias estadísticamente significativas en el número de años que llevan realizando botellón según su nivel de consumo de alcohol ( $\mathrm{V}: \mathrm{F}_{(3,954)}=8.20, \mathrm{MCE}=4.69$, $\left.p<0.001 ; M: F_{(3.1319)}=6.28, M C E=5.83, p<0.001\right)$. Tras realizar las pruebas a posteriori (Tukey), los resultados para los varones indican que sólo existen diferencias

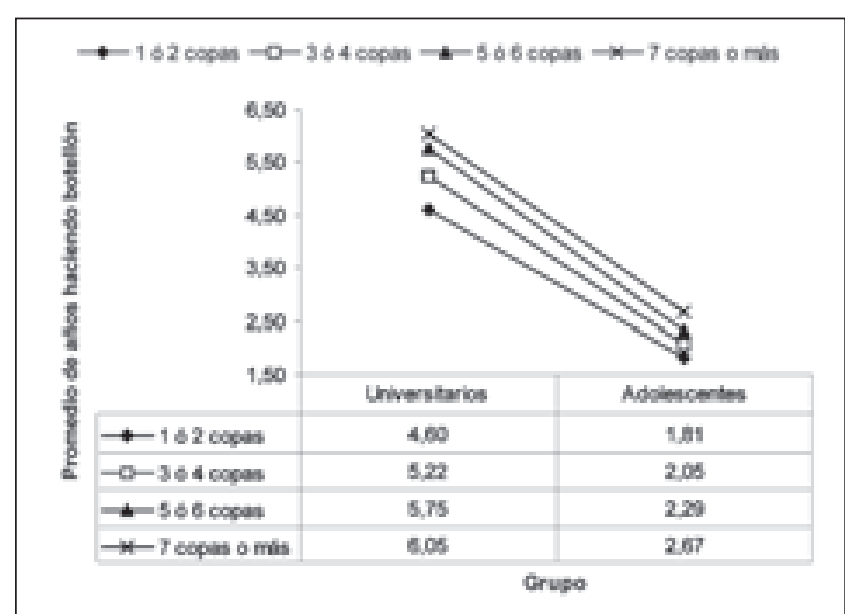

Figura 2. Gráfica para la interacción entre las variables Grupo y Consumo de alcohol

estadísticamente significativas entre el nivel más alto (7 copas o más) y todos los demás, mientras que para las mujeres las diferencias estadísticamente significativas se encuentran entre los dos niveles de consumo más bajo (1-2 copas, 3-4 copas) y los dos más altos (5-6 copas, 7 copas o más). Es decir, en general se observa que, tanto en varones como en mujeres, quienes más consumen son quienes más tiempo llevan haciendo botellón.

Pero además, en todos los niveles de consumo, el número de años que llevan haciendo botellón las mujeres frente a los varones es significativamente mayor ( $1-2$ copas: $t_{(205)}=2.06, p<0.041 ; 3-4$ copas: $\mathrm{t}_{(487.64)}=4.38, p<0.001 ; 5-6$ copas: $\mathrm{t}_{(694.18)}=5.57$, $\mathrm{p}<0.001 ; 7$ copas o más: $\left.\mathrm{t}_{(574)}=2.80, \mathrm{p}<0.005\right)$. 

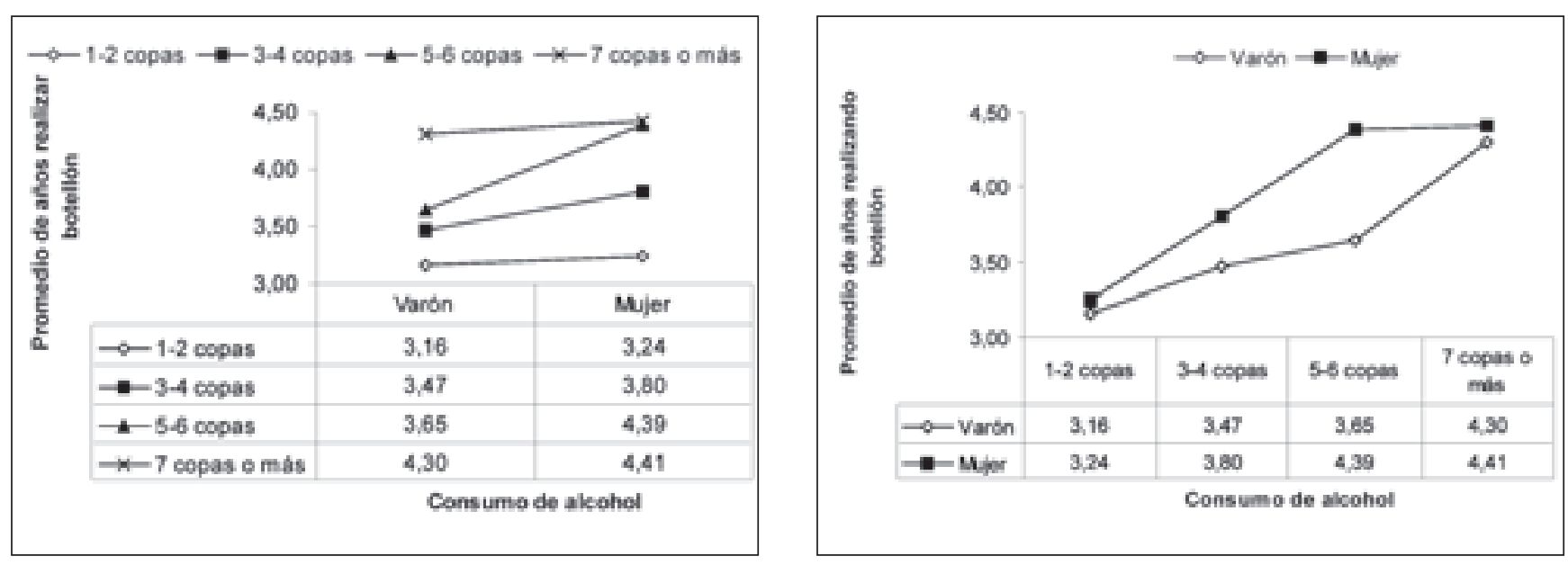

Figura 3. Gráficas para la interacción entre las variables Sexo y Consumo de alcohol

\section{Intervalo temporal de la conducta}

La hora de llegada al lugar donde se realiza boteIlón se sitúa entre las $22.40 \mathrm{~h}$. y la $1 \mathrm{~h}$. de la madrugada (Media=23.45h.). En cuanto a la hora a la que suelen abandonarla, la mayoría lo hace entre la 1h. y las $4.30 \mathrm{~h}$. de la madrugada (Media=3h.).

Se observan diferencias estadísticamente significativas en la hora de llegada al lugar donde se realiza el botellón en función del grupo $\left(F_{(1,2690)}=163.9\right.$, $M C E=1.83, p<0.001)$ y del sexo $\left(F_{(1,2690)}=13.99\right.$, $M C E=1.83, p<0.001)$. Los adolescentes llegan casi una hora antes (Media=23.30h.) al botellón que los universitarios (Media=00.15h.), y las mujeres Ilegan más tarde (Media=23.45h.) que los varones (Media=23.15h.). Sin embargo, no existen diferencias estadísticamente significativas en la hora a la que suelen abandonar el lugar donde están realizando el botellón (Media=3h.).

El número de horas durante las que realizan boteIlón difiere significativamente en función del grupo $\left(F_{(1,1903)}=33.69, M C E=1.27, p<0.001\right)$ y del consumo de alcohol $\left(F_{(3,1903)}=8.27, M C E=1.27, p<0.001\right)$. Concretamente, los adolescentes están como promedio más tiempo en el botellón ( 2.45 horas) que los universitarios (2.30 horas). Las pruebas a posteriori realizadas para los distintos niveles de consumo de alcohol (Games-Howell) muestran que no hay diferencias en el número de horas durante las que realizan botellón entre los de menor consumo (1-2 copas: Media=2.15 horas.; 3-4 copas; Media=2.20 horas), ni entre los de más consumo (5-6 copas: Media=2.35 horas; 7 ó más copas: Media $=2.40$ horas). Sin embargo, sí existen diferencias en los demás casos. Es decir, los que más consumen están más tiempo en el lugar donde se realiza el botellón que los que menos consumen.

\section{DISCUSIÓN}

A nivel general, la muestra analizada presenta un índice de iniciados en el consumo de alcohol similar al indicado en los informes publicados por el PNSD (2005, 2006). Tan sólo la edad de inicio en el consumo es ligeramente inferior, pudiendo deberse a que existe una diferencia de tres años en la recogida de información.

El porcentaje de adolescentes de 14 a 18 años que hacen botellón en la ciudad de Valencia supone un notable incremento si se compara con los datos generales que Huguet (2007) ofrece para toda la Comunidad Valenciana. Estos resultados son plenamente admisibles, si se tiene en cuenta que un muestreo de toda la Comunidad Valenciana implica la inclusión de muchos municipios de menor población en los que la presencia del botellón es mínima o inexistente.

Además, se trata de una conducta que va generalizándose entre los jóvenes conforme aumenta su edad, aunque no deja de ser relevante que más de la mitad de los adolescentes entrevistados de 15 a 17 años afirmen que ya están realizando botellón (15 años $=56.2 \% ; 16$ años $=68.9$ y 17 años $=77.2 \%$; Datos extraídos de Cortés, 2006). Esta vinculación creciente cuestiona algunas de las investigaciones descritas en la introducción que defienden que se trata de una opción de ocio minoritaria y limitada a jóvenes de mayor edad-universitarios-.

Asimismo, se confirma que no existen diferencias en función del sexo en el porcentaje de jóvenes que realizan botellón, independientemente de su edad. Hasta el momento se hablaba de una práctica más generalizada entre varones (Calafat et al., 2005), que sólo entre los más jóvenes parecía equipararse (GIESyT, 2001, 2002; Navarrete, 2004). El resultado obtenido en este trabajo reajusta esta consideración al 
haber evaluado la incidencia del botellón desvinculándola de las zonas concretas donde se realiza la conducta. Es probable que en los estudios de campo se encontrara una mayor presencia de varones debido al tipo de zonas de botellón seleccionadas, lo que habría llevado a obtener resultados parciales, no representativos del conjunto de jóvenes que hacen botellón.

Un dato importante a considerar a la hora de delimitar el momento óptimo para iniciar una actuación preventiva dirigida a paliar el fenómeno del botellón, es el descenso en la edad de inicio de esta práctica entre los adolescentes. Estamos ante una población que empieza a hacer botellón cada vez más pronto, lo que confirma las sospechas de Navarrete (2004), y de la cual puede afirmarse que suele hacer coincidir el inicio en el consumo de alcohol con la propia realización del botellón. Esta tendencia de los adolescentes no se aprecia entre los universitarios, los cuales solían iniciarse en el consumo de alcohol un año antes de empezar la práctica del botellón.

Esta situación alerta sobre la existencia de posibles consumos de riesgo, sobre todo si se tiene en cuenta la cantidad de alcohol que afirman ingerir los adolescentes durante el botellón, la cual se equipara en número de consumiciones con la de los universitarios. Puede afirmarse que se da una clara similitud entre la práctica europea que el Informe Alcohol in Europe denomina binge drinking y el nivel de consumo de los jóvenes durante el botellón, independientemente de su edad.

Al calcular un aspecto no conocido hasta el momento -los gramos de alcohol que ingieren los jóvenes, teniendo en cuenta la cantidad y el tipo de alcohol que dicen consumir-, los datos obtenidos sitúan esta muestra por encima de lo esperable. En el Informe Europeo, se equipara el consumo de alcohol de los jóvenes españoles con el del resto de países del sur de Europa -40gr. de alcohol- (Anderson y Baumberg, 2006). Pero los datos obtenidos en la presente investigación informan de una ingesta media de 5.3 consumiciones, lo que equivale a unos $50 \mathrm{gr}$. de alcohol, si se trata de bebidas de graduación baja, pudiendo llegar a unos $100 \mathrm{gr}$. si el consumo es de destilados de alta graduación. No hay que perder de vista que este consumo se realiza en un intervalo inferior a 3 horas, por lo que las tasas de alcohol en sangre que se alcanzan son elevadas.

Un dato esperable (Anderson y Baumberg, 2006; GIESyT, 2001; Navarrete, 2004) y confirmado en esta investigación es que continúan siendo los varones de todas las edades quienes consumen más cantidad de alcohol en cada sesión de botellón. Esto justifica el que las mujeres, para llegar al mismo nivel de consumo que un varón, requieran de más tiempo. Queda patente que son los varones quienes realizan un consumo más agresivo desde el inicio.
También se confirma el predominio de bebidas de alta graduación conforme se incrementa la edad -tal como apuntaban GIESyT (2001) y Navarrete (2004)-, pero no puede dejarse de lado que, sobre todo entre los adolescentes, siguen teniendo una importante presencia las bebidas de graduación baja y media.

Un aspecto no considerado hasta el momento y que es importante resaltar es el de la radicalización que se observa en el consumo por parte de las mujeres adolescentes, quienes ingieren bebidas de alta graduación al mismo nivel que los varones de su edad. Mientras que las mujeres universitarias se caracterizan por consumir en menor medida destilados de alta graduación en comparación con los varones, las mujeres adolescentes han equiparado su consumo. Por este motivo, aunque en edades superiores continúan siendo los varones quienes consumen más cantidad de alcohol y de una graduación superior, entre los adolescentes sólo se mantienen las diferencias entre sexos en la cantidad consumida.

El número de borracheras que dicen haber experimentado durante el último mes, aproximadamente una tercera parte de los jóvenes que hacen botellón, es similar al enunciado en los estudios de campo (Navarrete, 2004). Sin embargo, si se considera el grado de premeditación para alcanzar la embriaguez, se observa cómo entre los universitarios, los varones son más conscientes de querer llegar a ese estado, mientras que en el caso de las mujeres es una consecuencia imprevisible no relacionada con una planificación previa. Sorprende en este caso que los adolescentes muestren una conducta más extrema, ya que es mayor el porcentaje de varones y mujeres que tienen como objetivo llegar a la borrachera.

Si se vincula el consumo de alcohol tan elevado que se realiza en poco tiempo con la percepción subjetiva de llegar a la intoxicación se obtiene un primer indicador de la baja percepción de riesgo que tienen muchos de estos jóvenes. Es cierto, tal como apuntan algunos estudios (Crews et. al., 2000; White, Ghia, Levin y Swartzwelder, 2000) que la reducida susceptibilidad de los adolescentes a los efectos sedativos y a la incoordinación motora, permite comprender que alcancen concentraciones de alcohol en sangre elevadas sin llegar a experimentar una gran incapacitación. A pesar de ello, los jóvenes entrevistados llegan a consumir cantidades de alcohol tan altas que puede cuestionarse el que un porcentaje considerable de ellos niegue experimentar efectos propios de una intoxicación.

En cuanto a los índices de regularidad se confirma que se trata de una práctica de fin de semana (GIESyT, 2001) que abarca el intervalo jueves-sábado en el caso de la gente más adulta y el intervalo viernesdomingo en el caso de los más jóvenes, siendo los días de mayor asistencia los viernes y sábados. Pero 
además, la información sobre la frecuencia mensual añadida en esta investigación permite concluir que aunque con un incremento notorio en fechas vacacionales, esta conducta se realiza a lo largo de todo el año. De hecho, se observa que la mayor parte de adolescentes y universitarios la llevan a cabo durante seis o más meses, siendo amplio el porcentaje de ellos que la hacen todos los meses. Esta información permite hacer hincapié en que se trata de una conducta repetitiva, no ocasional, ni esporádica.

Al cruzar la información sobre regularidad de la conducta con la de la ingesta de alcohol realizada se aprecian diferentes modalidades de jóvenes que hacen botellón que hasta el momento eran desconocidos. Por una parte, aquellos que lo hacen durante menos años, meses y días a la semana y que realizan un consumo inferior a la media. Por otra parte, aproximadamente el 20\% de jóvenes que practican botellón durante más meses - 8 o más-, más veces a la semana -2 ó 3-, que consumen mayor cantidad de alcohol -superior a la media- y que lo están realizando durante más años -aproximadamente 3 años-.

Atendiendo por tanto al primero de los objetivos marcados en este trabajo -concretar las características que definen la población que hace botellón-, puede concluirse que nos situamos ante una conducta nocturna -de 23.45h. a 3h de la madrugada-; que tiene lugar durante el fin de semana -de jueves a domingo; que realizan de manera regular tanto mujeres como varones; desde los 14 años hasta los 25 años y en menor medida hasta los 30 años; que cada vez aparece a edades más tempranas coincidiendo con el inicio en el consumo de alcohol; en la que está presente un consumo elevado de alcohol -superior en el caso de los varones, y equiparable entre adolescentes y universitarios- aunque con diferencias en el tipo de alcohol que consumen. Concretamente, destaca el predominio de los destilados de alta graduación, pero sin dejar de considerar, sobre todo entre los más jóvenes, el lugar que ocupan las bebidas de graduación baja y media.

Además, es importante tener presente la radicalización que se observa en la conducta de consumo. Por una parte, las adolescentes muestran un patrón de ingesta similar al de los varones de su edad, igualándose en el tipo de alcohol consumido aunque no en la cantidad. Por otra parte, destaca el reconocimiento por parte de los adolescentes de buscar conscientemente la borrachera. Además, no puede obviarse que son muchos los jóvenes no conscientes de haber alcanzado un estado de intoxicación, a pesar de presentar un consumo superior a $50 \mathrm{gr}$. de alcohol, lo que muestra una vinculación entre el consumo realizado y una falsa percepción de control.

A partir de estas características, y atendiendo al segundo de los objetivos del trabajo, pueden extraer- se algunas orientaciones que sería adecuado tener en cuenta en la planificación futura de alternativas dirigidas a reducir la incidencia de esta conducta.

Los datos apoyan la necesidad de considerar dentro del fenómeno del botellón los aspectos de salud al mismo nivel que los aspectos sociales, ya que la mayoría de alternativas aplicadas para paliar esta conducta - p.e. presión policial, botellódromos, baldeo de calles, control de ruido...- prioriza la problemática social. Las cuestiones de salud suelen tener un abordaje indirecto y limitado al control externo, intentando impedir el acceso a la sustancia de diferentes maneras -control sobre establecimientos, control sobre la venta, etc.

Además, debe pensarse en alternativas diferenciadas de cara a reducir la importancia de esta asociación consumo de alcohol-ocio, ya que los datos alertan de la diversidad y heterogeneidad de jóvenes que están realizando esta práctica. Hay que situarse por tanto ante la planificación de alternativas dirigidas a diferentes colectivos: jóvenes que no se han iniciado, jóvenes que están realizando los primeros contactos con la práctica del botellón-consumo de alcohol y jóvenes que muestran una conducta más consolidada. La mayoría de las alternativas diseñadas y aplicadas hasta el momento para paliar el fenómeno del botellón -medidas judiciales, botellódromos, sustitución de la actividad de botellón por otras diferentes como deportes, lecturas, aventura, etc.-, no diferencian a los jóvenes en cuanto a su nivel de implicación en esta conducta.

Puede concluirse que se está descuidando la planificación de una prevención selectiva dirigida a los jóvenes que realizan esta práctica de manera regular, asociándolo a un alto nivel de consumo y en los que además el botellón parece ser "su único medio de ocio", dada la frecuencia con que lo realizan. En estos casos el control externo, aunque importante, no va a ser suficiente. Se requiere de un mayor nivel de intervención sobre aspectos personales, como su falsa percepción sobre los efectos que se experimentan con los niveles de consumo que están alcanzando.

Tampoco puede dejarse de lado un aspecto muy poco considerado en estos momentos: el nivel de motivación de esos jóvenes hacia el cambio en su conducta de botellón. Muchos de ellos no son conscientes de las consecuencias de su conducta -las tienen idealizadas- y por tanto no se plantean iniciar ningún cambio. En estos casos, todas las medidas externas -limitación de la venta de alcohol, control policial...- van a tener una utilidad limitada, al provocar estrategias de escape para poder continuar ejerciendo la conducta.

Pero no sólo debe focalizarse en los jóvenes el conseguir una mayor concienciación sobre las con- 
secuencias derivadas del botellón, ya que ésta se encuentra influida por la visión que sobre este fenómeno manifiesta la sociedad, y de manera más directa la gente que les rodea -padres, amigos, referentes importantes, etc.

Es necesario intervenir sobre la permisividad que muestra tanto la sociedad como los referentes importantes para el sujeto, hacia el consumo de alcohol juvenil y hacia la normalidad con la que se ve que el ocio de los jóvenes se lleve a cabo durante la noche. Respecto al consumo, es necesario dar a conocer la información real sobre los actuales patrones juveniles -muy diferentes a los de hace años-, lo que permitirá cuestionar que se trata de una conducta "normal" que todos han hecho alguna vez, y que no tiene consecuencias. Existen estudios, poco conocidos por el público general (García-Moreno et al., 2004; Tapert y Brown, 1999), que demuestran que los jóvenes son más vulnerables a algunas alteraciones cognitivas producidas por el consumo de alcohol, como alteraciones de memoria y aprendizaje.

En lo referente al ocio nocturno, sería adecuado plantear una reflexión acerca de si las alternativas psicosociales de ocio, que tienen que formar parte del paquete integrado de medidas para paliar el botellón, deben ajustarse a los horarios actuales de ocio nocturno, o si se requiere de un replanteamiento no sólo del tipo de actividades a realizar -introducir un hábito de ocio que sea formativo-, sino también del momento en el que estas se llevan a cabo.

Esta última reflexión apunta un nuevo aspecto. La intervención no puede limitarse a paliar las consecuencias derivadas de realizar esta conducta, es lógico que se atienda también a los determinantes que favorecen tanto el inicio como la consolidación de la misma -p.e. la permisividad social aludida o los hábitos de ocio aprendidos en la infancia o la actitud y conducta de los padres ante la realización del botellón por parte de sus hijos...-.

En estos momentos no corresponde hacer una discusión detallada de la viabilidad de las alternativas puestas en marcha en España para hacer frente al botellón, ya que se requiere necesariamente del conocimiento de más áreas -antecedentes y consecuentes principalmente- que nos permitan conocer con mayor profundidad esta conducta. Por este motivo, emplazamos esta discusión para futuros trabajos.

\section{AGRADECIMIENTOS}

Los datos presentados en este trabajo forman parte de la investigación financiada por el Plan Nacional Sobre Drogas en la convocatoria 2003-2006.

\section{REFERENCIAS}

Aguilera, R. (2002). Generación botellón ¿Qué hay detrás del botellón? Madrid: Oberon.

Alternativajoven (2006a). Bebiendo junto al Guadiana: Estudio sobre el botellón en el paseo fluvial de Badajoz. Recuperado el 25 de enero de 2007, de www.alternativajoven.es

Alternativajoven (2006b). Bebiendo junto al Guadiana: Estudio sobre el botellón en el recinto ferial de Mérida. Recuperado el 25 de enero de 2007, de www.alternativajoven.es

Anderson, P. y Baumberg, B. (2006) Alcohol in Europe. London: Institute of Alcohol Studies.

Baigorri, A., Fernández, R. y GIESyT (2003). Botellón. Un conflicto postmoderno. Barcelona: Icaria.

Borrás, F. (2004). Análisis antropológico del tiempo de ocio de la juventud en la provincia de Alicante. Alicante: Diputación de Alicante, Universidad Miguel Hernández.

Calafat, A., Juan, M., Becoña, E., Castillo, A., Fernández, C., Franco, M., Pereiro, C. y Ros, M. (2005). El consumo de alcohol en la lógica del botellón. Adicciones, 17, 193-202.

Cortés, M. T. (2005, noviembre). Aspectos psicosociales del fenómeno del botellón: Pasado, presente y futuro. Ponencia invitada al VI Fórum. El botellón: Causas, Efectos y Alternativas, Palma de Mallorca.

Cortés, M. T. (2006, noviembre). Realidad del fenómeno botellón: ¿hacia donde vamos? Comunicación presentada en el 20 Congreso Sociedad, Familia y Drogas (FAD), Valencia.

Cortés, M. T., Espejo, B., Giménez, J. A., Mestre, M. V., Samper, P., Nácher, M. J. y Dolz, L. (2006, Julio) Peculiarities in high consumption of alcohol in youths. Is there a worldwide tendency?, Póster presentado en el 26th International Congress of Applied Psychology, Athens.

Cortés, M. T., Giménez, J. A., Nácher, M. J., Pascual, P., Vicéns, S. (2006, marzo) Características del fenómeno botellón en Valencia: ¿se corresponden las alternativas con las necesidades? Póster presentado en las XXXIII Jornadas Nacionales de Socidrogalcohol, Cáceres.

Crews, F.T., Braun, C. J., Hoplight, B., Switzer, R. C., Knapp, F. y Darin J. (2000). Binge Ethanol Consumption Causes Differential Brain Damage in Young Adolescent Rats Compared With Adult Rats. Alcoholism: Clinical \& Experimental Research. 24, 1712-1723.

Domínguez, S., Gómez, R., Jáñez, M. y Barrantes, C. (2001). La cultura del botellón. Recuperado el 9 de febrero de 2007, de http://www.aidex.es/observatorio/temas/ botellon/estudio/indicestbote.htm

Elzo, J., Laespada, M. T. y Pallarés, J. (2003). Más allá del botellón. Análisis socioantropológico del consumo de alcohol en los adolescentes y jóvenes de la Comunidad de Madrid. Madrid: Agencia Antidroga. Consejería de Sanidad. 
Espejo, B., Cortés, M. T., Giménez, J. A., Samper, P. y Pardo, F. J. (2006, julio). Different styles of life connected with the high consumption of alcohol in adolescence. Notions coming from parent and children. Póster presentado en el 26th International Congress of Applied Psychology, Athens.

García-Moreno, L. M., Capilla, A., García-Sánchez, O., Luque, J., Senderek, K., Conejo, N. M. y Arias, J. L. (2004). Alcohol tolerance in rats submitted to different periods of chronic and acute ethanol intake. Psicothema, 16, 211-216.

GIESyT (2001). El botellón en las ciudades de Badajoz, Cáceres, Mérida y Plasencia. Universidad de Extremadura. Consejería de Cultura y Patrimonio

GIESyT (2002). Avance de la Investigación Sociológica. Universidad de Extremadura

Giménez, J. A., Cortés, M. T., Nácher, M. J., Espejo, B. y Plaza, B. (2006, julio). Alternatives to stop street drinking in spanish youngsters. Similarities and differences with other countries." Póster presentado en el 26th International Congress of Applied Psychology, Athens.

Huguet, P. (2007). Uno de cada seis adolescentes valencianos practica el botellón los fines de semana. Las Provincias, 10/02/2007.

Navarrete Moreno, L. (2004). Juventud y drogas: 4 estudios sociológicos comparados. Madrid: Ilustre Colegio Nacional de Doctores y Licenciados en Ciencias Políticas y Sociología.

Nogués, A., Borrás, F., Segura, J. V., Ruiz, J. L. y Botella, F. (2004, septiembre). Carnavalización juvenil del fin de semana: el botellón. Comunicación presentada en el VIII Congreso Nacional de Sociología, Alicante.

Pardo, F. J., Plaza, B., Giménez, J. A. y Cortés, M. T. (2005, noviembre). ¿Qué repercusión tiene actualmente el botellón en los medios de comunicación valencianos? Póster presentado en el Congreso de Estudiantes de Elche, Elche.

Plan Nacional sobre Drogas (2005). Observatorio Español sobre drogodependencias. Encuesta escolar 2004. Madrid: Delegación del Gobierno para el Plan Nacional sobre Drogas. Ministerio de Sanidad y Consumo.

Plan Nacional sobre Drogas (2006). Observatorio Español sobre drogodependencias. Encuesta domiciliaria 2005-2006 (adelanto). Recuperado el 8 de enero de 2007, de http://www.pnsd.msc.es/Categoria2/observa/ pdf/Domiciliaria2005-2006.pdf

Plaza, B. (2006). El fenómeno botellón en los principales medios de comunicación de la Comunidad Valenciana. Trabajo para la obtención del Diploma de Estudios Avanzados. (Dir. M.T. Cortés). Universitat de Valéncia.

Plaza, B., Pardo, F., Cortés, M. T., Samper, P., Dolz, L y Martínez, A. (2006, marzo). Cómo trata la prensa valenciana el fenómeno botellón: necesidad de superar una visión restrictiva. Póster presentado en las XXXIII Jornadas Nacionales de Socidrogalcohol, Cáceres.

Rodríguez-Martos, A. (2003). Ley del Botellón versus Ley de la Bota de Vino. Adicciones, 15, 3-5.

Sánchez, M. J. y Rubio, L. (2001). El botellón en la ciudad de Madrid. Estudio sociológico. Trabajo fin de carrera. Universidad Autónoma de Madrid.

Tapert. S. F. y Brown S. A. (1999). Neuropsychological correlates of adolescent substance abuse: four-year outcomes. Journal of the International Neuropsychological Society, 5, 481-93.

White, A. M., Ghia, A. J., Levin, E. D. y Swartzwelder, H. S. (2000). Binge pattern alcohol exposure: differential impact on subsequent responsiveness to alcohol. Alcoholism. Clinical Experimental Research, 24, 1251-1256. 